\title{
Neue Möglichkeiten zur technischen Unterstützung bei der Ausbildung an ERP-Systemen
}

\section{Adaptive Lernkonzepte durch AUM und Enterprise Tomo- graphie}

\author{
Liane Haak, Dirk Peters, Jan Aalmink \\ Department für Informatik, Abteilung Wirtschaftsinformatik I, \\ Carl von Ossietzky Universität Oldenburg \\ Ammerländer Heerstr. 114-118, 26129 Oldenburg \\ \{haak|peters|ent.tomo\}@wi-ol.de
}

\section{Herausforderungen in der Lehre}

Konfrontiert mit einem internationalen Bildungsmarkt, mit neuen konkurrierenden und einer wachsenden Anzahl von Angeboten, verbunden mit der fast unbeschränkten Mobilität der Lernenden und der Bereitschaft sich ständig weiterzubilden, steht unser Bildungswesen vor einer großen Herausforderung. Davon sind auch Bildungseinrichtungen wie Hochschulen und Universitäten betroffen. Studienabschlüsse sind kein „Selbstzweck“, sondern unterliegen steigenden Anforderungen, wie z. B. denen der Industrie nach kürzeren Studienzeiten bei gleichzeitiger Orientierung an den Problemen der Praxis. Besonders in stark anwendungsorientierten Studiengängen wie der Wirtschaftsinformatik ist das der Fall (Rautenstrauch 2008; Freyburger und Lehmann 2008). Der Bachelor, als erster berufsbefähigender Abschluss, ist dafür ein gutes Beispiel.

In diesem Markt müssen Hochschulen und Universitäten sich praktischlaufend neu erfinden sowie Schwerpunkte in der Ausbildung setzen, um sich auf dem Bildungsmarkt abzuheben, auf europäischer und nationaler Ebene zu kooperieren und um wettbewerbsfähige Curricula anbieten zu können. Darüber hinaus gilt es den Bedürfnissen der Lernenden zur Unterstützung des lebenslangen Lernprozesses in verschiedenen Phasen (z. B. auch in der Weiterbildung, nicht nur in der 
Erstausbildung) zu entsprechen und Konzepte mit flexiblem Zugang unter Nutzung modernster Technologien anzubieten.

Aktuelle Informations- und Kommunikationstechnologien bieten hierzu einige Hilfestellungen, um den Lemprozess zu unterstützen und reichen vom Lernmanagement bis hin zum ortsunabhängigen E-Learning. Forschungen im Bereich Technology Enhanced Learning sind daher seit Jahren Schwerpunkt in der Förderlandschaft sowohl auf nationaler Ebene ${ }^{1}$ als auch in der Europäischen Union ${ }^{2}$. Sie fokussieren dabei im Gegensatz zum E-Learning auch auf die technologische Unterstützung von pädagogischen Ansätzen. Beispiele hierfür finden sich in dem vom 6 . Europäischen Rahmenprogramm geförderten Networks of Excellence „PROLEARN”3 und „Kaleidoscope”4, welche u. a. die Aufgabe haben, Forschungen und Zielsetzungen in diesem Umfeld zu konkretisieren und zu steuern.

\subsection{Defizitäre Ausbildung an ERP-Systemen}

Wenn in der heutigen Zeit über den Einsatz und die Funktionalität betrieblicher Anwendungssysteme gesprochen wird, ist der Fokus der Betrachtung meist auf die Integration möglichst vieler Unternehmensbereiche gerichtet. Das Bestreben heutiger betrieblicher Anwendungssysteme - insbesondere ERP-Systeme - ist daher die Ausrichtung auf ein Allround-Produkt, das im besten Fall die gesamte Funktionalität einer Unternehmung abdeckt. Vordergründig geht es also um die Integration von Daten, Funktionen und Prozessen aus allen Bereichen innerhalb einer Unternehmung.

Ein kritischer Punkt dieser Entwicklung ist die Konfrontation des Anwenders mit einer immer größer werdenden Komplexität der Systeme. Folglich steigt auch die Anzahl an möglichen Navigationsmöglichkeiten in einem System kontinuierlich an, so dass es für den Anwender immer schwieriger wird, den Umgang mit diesen Systemen zu erlernen und diese Systeme später effizient im täglichen Betrieb einzusetzen. Aber Unternehmen fordern genau diese Ausbildung an großen betrieblichen Anwendungen, wie z. B. ERP-Systemen, von den heutigen Absolventen, denn sie haben ein großes Potential für die Illustration, Visualisierung und Simulation von Geschäfts- und Entscheidungsunterstützungsprozessen (Ask et al. 2008).

Ein weiteres Problem besteht in dem Mangel an weitreichenden, didaktisch aufbereiteten Schulungsmaterialen in Form von Fallstudien u. a.. Häufig existieren zwar ausgiebige theoretische Einführungen in die Benutzung einer Software, diese enthalten jedoch insgesamt zu wenig praktische Anteile. Materialien zur Schulung an den Systemen selbst, basieren wiederum häufig auf Trainingsmaterialien der

\footnotetext{
1 z. B. http://www.bmbf.de/de/equalification.php

2 z. B. http://cordis.europa.eu/fp7/ict/telearn-digicult/telearn_en.html

${ }^{3}$ http://www.prolearn-project.org/

${ }^{4}$ http://www.noe-kaleidoscope.org/pub/
} 
Hersteller, die eher darauf abzielen, den (späteren) Anwender mit einzelnen Funktionen oder Modulen der Software vertraut zu machen (z. B. Fallstudien des International Demonstration and Education Systems (IDES) ${ }^{5}$ der SAP®AG oder der Cronus $\mathrm{AG}^{6}$ von Microsoft ${ }^{\circledR}$ Dynamics). Zwar werden diese Schulungsmaßnahmen durch Institutionen wie den University Competence Centern (UCC) der SAP ${ }^{\circledR} A G$ oder dem Partner Netzwerk von Microsoft ${ }^{\circledR}$ umfangreich technisch unterstützt (Courant 2008), doch fehlt nach wie vor häufig die Prozesssicht aufgrund des primären Fokus auf die Systemfunktionalität. Studierenden fällt es daher schwer, die Hintergründe, warum sie bestimmt Transaktionen ausführen sollen, zu verstehen.

Weiterhin finden didaktische Herangehensweisen, wie beispielsweise Wissenskontrollen, Reflektionen über das zuvor gelernte oder Ideen zu Gruppen- und Individualarbeiten selten ausreichendes Augenmerk (Hans et al. 2008). Der strategische Aspekt hinter vielen Entscheidungen bleibt zunächst oft unberücksichtigt und daher für den Lernenden nicht nachvollziehbar.

\subsection{Der Lernende im Mittelpunkt}

Zielsetzung moderner Lehr- und Lernkonzepte muss es sein, den Lernenden in den Mittelpunkt zu stellen, und neuste Technologien zur Unterstützung der Ausbildung zu nutzen (Brehm et al. 2009). Bildungsangebote der Zukunft sind als Services zu verstehen, dessen Sinn es sein wird, gezieltes Wissen mit der bestmöglichen technischen Unterstützung zu vermitteln. Dem Bedürfnis nach ortsunabhängigem Lernen und neu konzipierte Lernumgebungen ist dabei ebenso Rechnung zu tragen, wie der zunehmenden Vernetzung der Lernenden und Lehrenden durch die Einbeziehung von interaktiven Angeboten und Web 2.0 Applikationen, wie z. B. Communities, Blogs etc. Zu letzterem existieren bereits Ansätze die sich an anderen Domänen orientieren und davon lernen (z. B. Game-Based Learning (St. Pierre 2007)). Ziel dieser Ansätze ist es herauszufinden, wie Lernspiele konzipiert sein müssen, damit gerne gelernt wird, aber auch wie von etablierten Spielkonzepten (wie z. B. World of Warcraft) gelernt werden kann, wie Communities of Practice eigentlich aufgebaut sein müssen, damit sie überhaupt funktionieren. Letztlich sind auch Gender Aspekte zu berücksichtigen, da es häufig Unterschiede in den Lernweisen der verschiedenen Geschlechter gibt. Deren Betrachtung kann besonders E-Learning-Konzepten zum Erfolg verhelfen (Wolf 2007).

Im Mittelpunkt dieses Beitrags steht die Vorstellung eines neuen Ansatzes, der zur Schulung an ERP-Systemen konzipiert wurde. Ziel ist es, Technologien zur Dokumentation des Benutzerverhaltens zu nutzen, um den Lernfortschritt zu dokumentieren. Dadurch wird es möglich, den Lernenden zu lenken und in seinem

\footnotetext{
5 http://help.sap.com/saphelp_46c/helpdata/DE/af/fc4f35dfe82578e10000009b38f839/ frameset.htm

${ }^{6}$ http://www.microsoft.com/germany/bildung/infopool/mbsaa.mspx
} 
Lernprozess individuell zu unterstützen. Zusätzlich bekommt der Lehrende eine Rückmeldung über den Fortschritt im Lernprozess und über damit einhergehende, auftretende Schwierigkeiten. Dies ist gleichzeitig eine Vorbedingung, um mittelfristig die Ausbildung an großen betrieblichen Anwendungssystemen durch ELearning ermöglichen zu können. Dieser Beitrag behandelt somit das adaptive, angepasste Lernen des Individuums unter Einsatz modernster Informationstechnologie (IT).

\section{Technische Möglichkeiten zur Analyse des Benutzerverhaltens in ERP-Systemen}

In Bezug auf die neuen technischen Möglichkeiten zur Analyse des Benutzerverhaltens in betrieblichen Anwendungssystemen im Allgemeinen und ERP-Systemen im Speziellen existieren zwei Ansätze, die im folgenden etwas näher betrachtet werden sollen. Im Vordergrund steht dabei das übergeordnete Ziel, anhand der durchgeführten Tätigkeiten oder Arbeitsschritte eines Anwenders an einem System, Rückschlüsse auf seinen Wissensstand zu schließen. Daraus werden Verhaltensmuster abgeleitet, auf deren Basis dem Anwender dann wiederum Anweisungen oder Hilfestellungen zur Bewältigung bestimmter Aufgaben geliefert werden können. Im folgenden Abschnitt wird zunächst der Ansatz des Application Usage Mining erläutert, wonach dann die Vorstellung des Ansatzes der Enterprise Tomographie und deren gemeinsame Verwendung folgt.

\subsection{Application Usage Mining (AUM)}

Beim AUM handelt es sich um ein Verfahren zur Rekonstruktion von IstWorkflow-Modellen. Dabei bezieht der Ansatz seine Informationen aus Protokollund Trace-Daten, um Muster von Workflow-Instanzen zu erkennen und anschlieBend zu analysieren. Das Verfahren kann zur Ermittlung des Benutzerverhaltens in einem betrieblichen Anwendungssystem verwendet werden, indem die Bewegungen des Anwenders in einem System protokolliert werden (Kassem 2007). Der Ansatz basiert weitestgehend auf dem anerkannten Verfahren des Web Usage Mining (WUM). Die signifikanten Unterschiede beider Ansätze resultieren aus ihrem Anwendungsgebiet (AUM für betriebliche Anwendungssysteme und WUM für Web Applikationen) und werden nachfolgend in Tabelle 1 gegenübergestellt. 
Tabelle 1: Vergleich zwischen WUM und AUM (Kassem 2003)

\begin{tabular}{|l|l|l|}
\hline Attribute & Web Applikation & $\begin{array}{l}\text { Betriebliches } \\
\text { Anwendungssystem }\end{array}$ \\
\hline Systemzugriff & Anonym & $\begin{array}{l}\text { Anwender wird eindeutig identi- } \\
\text { fiziert }\end{array}$ \\
\hline Autorisierung & $\begin{array}{l}\text { keine Autorisierung } \\
\text { erforderlich }\end{array}$ & $\begin{array}{l}\text { Anwender verfügt über bestimm- } \\
\text { te Berechtigungen gemäß seiner } \\
\text { Funktion im Unternehmen }\end{array}$ \\
\hline Protokoll & Standard HTTP & keine Standards \\
\hline Software & $\begin{array}{l}\text { Web Applikation - meist } \\
\text { basierend auf HTML }\end{array}$ & $\begin{array}{l}\text { Unterschiedliche Softwareplatt- } \\
\text { formen }\end{array}$ \\
\hline Anwenderverhalten & frei & $\begin{array}{l}\text { Ausführung vordefinierter Auf- } \\
\text { gaben und } \\
\text { Geschäftsprozesse }\end{array}$ \\
\hline $\begin{array}{l}\text { Anwendungs- } \\
\text { gegenstand }\end{array}$ & nicht definiert & $\begin{array}{l}\text { Optimale Durchführung von } \\
\text { Aufgaben und } \\
\text { Geschäftsprozessen }\end{array}$ \\
\hline $\begin{array}{l}\text { Ziel der } \\
\text { Anwendung }\end{array}$ & $\begin{array}{l}\text { die Erreichung einer } \\
\text { möglichst hohen Anzahl } \\
\text { an potentiellen Kunden }\end{array}$ & $\begin{array}{l}\text { Effiziente Ausführung und Au- } \\
\text { tomatisierung von } \\
\text { Geschäftsprozessen }\end{array}$ \\
\hline
\end{tabular}

Ein wichtiger Punkt dabei ist, dass ein Anwender bei der Arbeit mit einem betrieblichen Anwendungssystem eindeutig identifiziert werden kann, während diese Eigenschaft beim WUM durch die Anonymität des Anwenders nicht auftritt. Auf diese Weise wird es möglich, individuelle Verhaltensmuster abzuleiten und konkret die Belange einzelner Anwender zu betrachten.

Eine Voraussetzung für die Verwendung des AUM-Ansatzes ist es, möglichst detaillierte Tracking-Informationen über die vom Anwender ausgeführten Tätigkeiten im System vorzuhalten. Der Aufbau einer Protokoll-Datei im SAP R/3System ist in Abbildung 1 dargestellt. Darin ist zu erkennen, dass im SAP-System eine Fülle von Informationen in Protokollen vorhanden ist, die eine genaue Analyse des Benutzerverhaltens ermöglicht. In dem Beispiel ist weiterhin zu sehen, welche Werte der Anwender über die grafische Benutzungsoberfläche des Systems eingegeben hat und es ist es möglich, konkrete Aussagen über die Dauer einzelner Schritte innerhalb von Transaktionen, ihre gesamte Aufrufhäufigkeit oder auftretende Fehlermeldungen zu erhalten. 


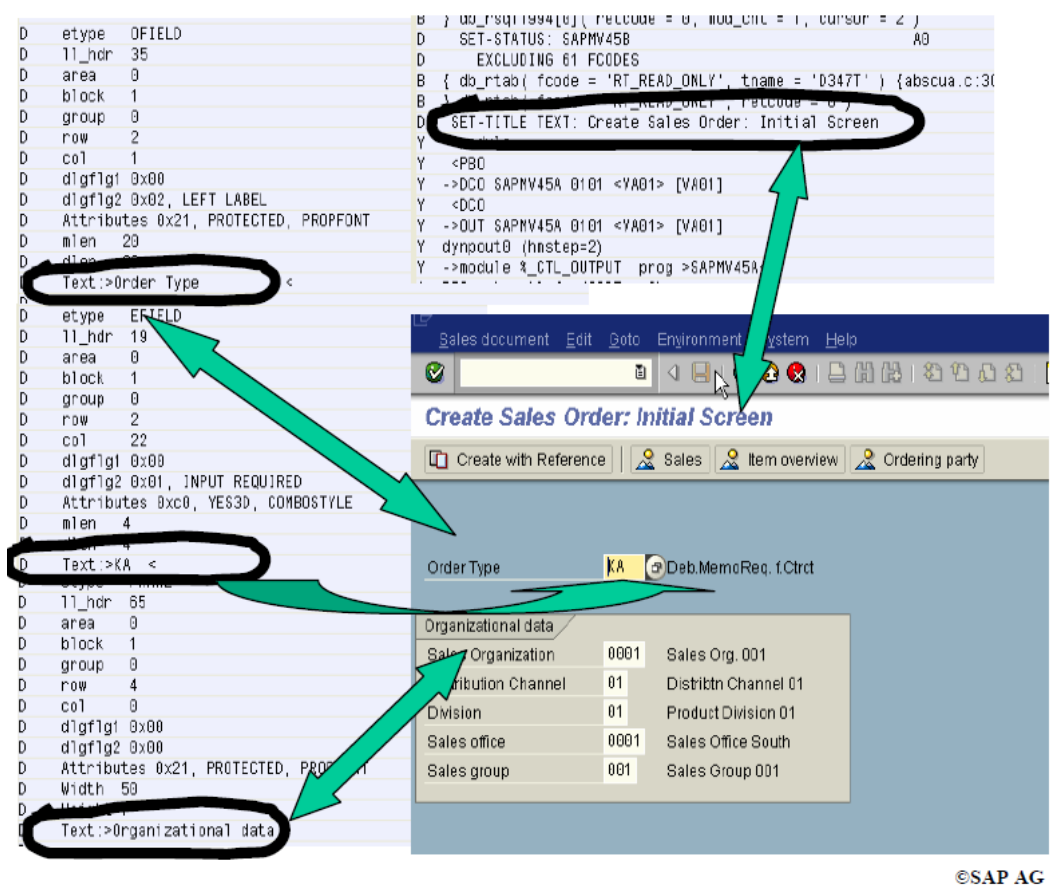

Abbildung 1: Aufbau einer Protokoll-Datei im SAP R/3

Neben dem AUM existiert noch das Verfahren der Enterprise Tomographie, welches in Kombination im späteren Konzept helfen wird, das Benutzerverhalten zu analysieren und damit den Lernfortschritt des Einzelnen zu steuern.

\subsection{Enterprise Tomographie}

Der Enterprise Tomograph (ET) ist ein Meta-Informationssystem, d. h. ein Informationssystem für ein betriebswirtschaftliches Informationssystem. Das Application Lifecycle Management wird mit dem ET in effizienter Weise unterstützt (Aalmink und Marx Gómez 2009). Alle relevanten Integrations- und Metadaten eines Informationssystems, als auch die verwalteten und verarbeiteten betriebswirtschaftlichen Daten über Customizing und Dokumentation sind über einen InMemory Index zugreifbar. Mit Hilfe einer Such-Oberfläche können Tomogramme des gescannten Datenuniversums instantan generiert werden. Wie in Abbildung 2 dargestellt, können zwischen zwei Zeitpunkten die Änderungen des Informationssystems und dessen Daten mit Hilfe der Tomogramme visualisiert werden (DeltaErmittlung). 


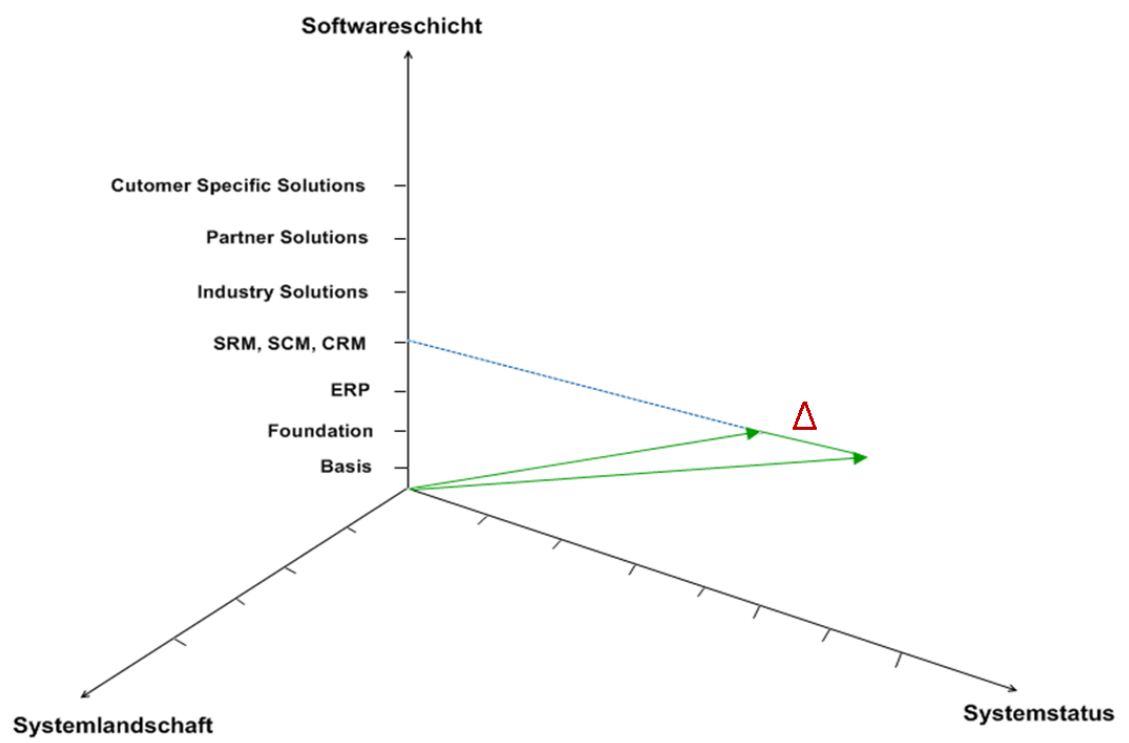

\section{Abbildung 2: Delta-Ermittlung im Enterprise Tomographen}

In Analogie zur medizinischen Apparativ-Diagnostik soll der ET dynamisch Tomogramme als Artefakte berechnen, welche eine semantische (Teil-)Sicht auf die gesamte Software-Landschaft repräsentieren. Veränderungen sollen ebenso in Tomogrammen repräsentiert und anhand dieser Fehlerpotenziale geortet werden, z. B. um wartungsrelevante Entscheidungen zu treffen. Der ET fungiert hier als Näherungsverfahren für die Fehlerlokalisierung (semi-automatische Fehlersuche).

Delta-Berechnungen sind grundsätzlich nicht neu und es existieren hierzu zahlreiche, individuell mit hohem Aufwand konstruierte Verfahren, sowohl in der Praxis als auch in der Wissenschaft. Der im Rahmen dieses Forschungsprojektes anvisierte Ansatz ist im Vergleich zu den individuellen Lösungen ein generischer Ansatz. Die Delta-Berechnung innerhalb eines Merkmalsraums soll zentral in einem generischen Algorithmus erfolgen, auf dessen Basis dann marktfähige Produkte ohne nennenswerten Mehraufwand kunden- bzw. problemspezifisch ausgeprägt werden können. Die Leistungspotenziale von Algorithmen aus der Bioinformatik, der bekannten Suchmaschinen und von den Columnaren InMemory-Datenbanken sollen bei der Delta-Ermittlung voll ausgeschöpft werden. Einerseits ermöglicht dieser generische Ansatz die Produktkonfiguration (Bereitstellung der Variantenvielfalt ohne Mehraufwand), andererseits hat jede Produktvariante im Inneren hochleistungsfähige Algorithmen vereint und integrativ aufeinander abgestimmt.

Die Einfachheit in der Individualisierung dieses Ansatzes ermöglicht die Lösung von vielen praxisrelevanten Fragestellungen und ist auch anwendbar im Rahmen von Informationssystem-Vergleichen, z. B. eines Produktivsystems mit einem Testsystem oder beim Einsatz im Umfeld von Lehr- und Lernumgebungen (zum Beispiel vor und nach der Durchführung einer Übungsaufgabe eines Lernen- 
den). Durch die Verknüpfung mit dem AUM-Ansatz entsteht dadurch ein effizientes System zur Lernfortschrittsverfolgung im Rahmen von Schulung an ERPSystemen.

\section{Konzept zur Lernfortschrittsverfolgung}

Welche Rolle spielen die Ansätze AUM und Enterprise Tomographie bei der Schaffung einer Lehr- und Lernumgebung? Wie bereits angesprochen, kann der AUM-Ansatz verwendet werden, um Verhaltensmuster eines Anwenders zu identifizieren oder mögliche Defizite eines Anwenders bei der Durchführung bestimmter Aufgaben am System aufzudecken. Das Ziel ist es, ein Lehr- und Lernsystem zu schaffen, welches im Rahmen von Curricula an Universitäten eingesetzt werden kann, um Studierenden den Umgang mit Anwendungssystemen (z. B. SAP® ERP) näher zu bringen, auf den individuellen Kenntnisstand des Einzelnen einzugehen und zu verbessern. Dabei steht die Bewältigung von Problemen wie heterogene Studierendengruppen, unterschiedliche Wissensvoraussetzungen und divergierende Lernverhaltensmustern im Vordergrund.

\subsection{Beispielszenario}

Um das angestrebte Konzept zu konkretisieren, erfolgt zunächst eine detaillierte Betrachtung der technischen Umsetzung der adaptiven Lernumgebung anhand eines Beispiels mit dessen Hilfe dargestellt wird, wie die Durchführung der Aufgabe „Bestellung aufnehmen“ aus Sicht des Anwenders aussehen könnte. Anhand eines vorgegebenen Pfades, wie er in Abbildung 3 in Form eines Petri-Netzes dargestellt ist, wird der optimale Lösungsweg festgelegt. Dieser umfasst im ersten Zustand $A$ die Aufnahme einer Bestellposition. Hier soll der lernende Anwender einen beliebigen Artikel auswählen, den ein Kunde bestellen möchte. Hat der Lernende eine Bestellposition ausgewählt, kann er die Bestellung im nächsten Zustand $B$ abschließen oder der Bestellung über den Zustand $C$ weitere Bestellpositionen hinzufügen, um erst danach zum Zustand $B$ zu gelangen und die Bestellung abzuschlieBen.

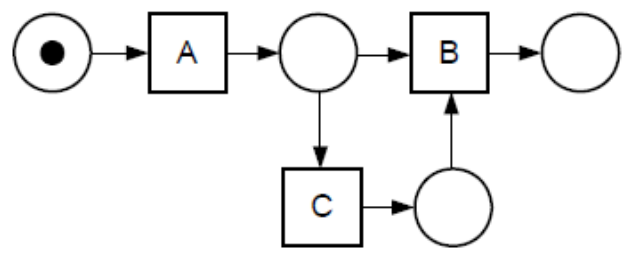

Abbildung 3: Petri-Netz zur SAP Transaktion „Bestellung aufnehmen“

Mit Hilfe des AUM-Ansatzes kann nun im nächsten Schritt analysiert werden, welche Zustände welcher Anwender mit welchen Eingabewerten erreicht hat. Es kön- 
nen Rückschlüsse auf die Verweildauer eines Anwenders in beliebigen Eingabemasken geschlossen werden, um darauf basierende Hilfestellungen anzubieten. Hat ein Anwender z. B. viel Zeit in Zustand $C$ verbracht und keine weiteren Bestellpositionen angelegt, ist anzunehmen, dass er die falsche Eingabemaske aufgerufen hat und die Bestellung ursprünglich direkt über Zustand $B$ abschließen wollte. Sprechen weitere Merkmale in den Protokoll-Dateien (z. B. mehrfaches Klicken auf den Abbruch-Button) für diese Annahme, kann dem Anwender das (erneute) Erlernen der Navigationsmöglichkeiten innerhalb des Systems nahegelegt werden, damit er diesen Fehler in Zukunft vermeiden kann.

\subsection{Enterprise Tomographie erweitert AUM-Ansatz}

Das Verständnis für Geschäftslogiken innerhalb betrieblicher Anwendungssysteme setzt die Vergegenwärtigung der darunterliegenden Integrationskonzepte voraus. Normalerweise wird dieses Integrationswissen auf Basis von Schulungsunterlagen in Kombination mit praktischen Übungen an Demo-Systemen erworben. Integrationskonzepte und ihre Verwendung sind jedoch schwer begreifbar und durch die existierenden, klassischen Lehr- und Lernmethoden nicht leicht zu vermitteln. Mit Hilfe des integrierten ET-Ansatzes kann die Phase der Ermittlung und Bewertung des Lernfortschritts automatisiert werden. Im Gegensatz zum AUM-Ansatz liefert der ET eine eher integrative Sicht auf die Lernumgebung. Während sich der AUMAnsatz sehr auf die konkrete technische Analyse des Benutzerverhaltens mit Hilfe von Protokoll- und Trace-Dateien konzentriert, verdeutlicht der ET die weitreichenden Auswirkungen einzelnen Transaktionen auf das Gesamtsystem. Es geht also darum, dem Lernenden die Integrationsaspekte betrieblicher Anwendungssysteme sichtbar zu machen.

Einen Überblick über das neue Lernszenario zeigt Abbildung 4: durch die Adaptive Learning-Umgebung erhält jeder Lernende einen ganzheitlichen Blick auf die dem System zugrundeliegenden Geschäftsprozesse. Abstrahierend wird das Modell eines Geschäftsprozesses als Graph mit genau einem Startpunkt und einem Endpunkt (vereinfachtes Petri-Netz) betrachtet (sog. Business Process Plattform). Jeder Knoten entspricht einem Konzept, d.h. einem Element einer Ontologie (zum Beispiel ein Fertigungsauftrag) mit entsprechenden typgleichen KonzeptInstanzen, und eine Kante repräsentiert eine transaktionale Abhängigkeit, also einen durchzuführenden Lernschritt. 


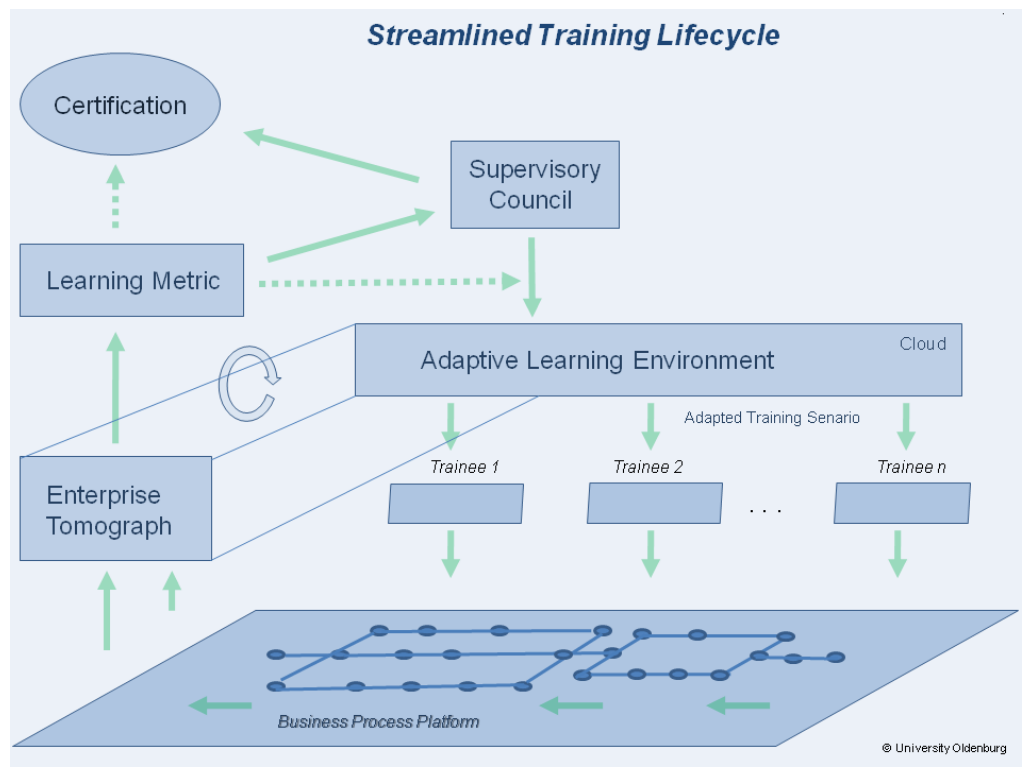

\section{Abbildung 4: Adaptive Lernumgebung auf Basis des Enterprise Tomographen}

Eine Warenbewegung eines Materials von Lagerort auf Fertigungsauftrag wäre in diesem Sinne also ein Lernschritt bzw. eine Transition in dem Graphen LagerortMaterial-Fertigungsauftrag. Die Knoten beinhalten jeweils 0-n Konzept-Instanzen, der Graph ist zweistufig zu betrachten. Die Abhängigkeitsbeziehungen zwischen den Konzepten entsprechen dem Vorgehensmodell der Lernenden (Prozesskette). Der Lernende (Trainee) versucht den Beispielgeschäftsprozess entsprechend dem Graphen durchzuführen. Dabei werden im ERP-System Konzept-Instanzen erzeugt, verändert oder gelöscht.Die Menge der veränderten, gelöschten oder erzeugten Konzept-Instanzen ist im Vergleich zu den unveränderten KonzeptInstanzen von der Kardinalität her sehr klein. Die Differenz kann der Enterprise Tomographie Algorithmus als Delta (Delta-Bäume) mit Hilfe von DeltaIndizierung effizient errechnen.

Da das Lern-Vorgehensmodell (die optimale Sequenz der vom Lernenden (Trainee) auszuführenden Prozessschritte) bekannt ist, ermöglicht das vom ET ermittelte Delta das Ziehen von semi-automatischen Rückschlüssen über den getätigten Lernfortschritt. Die Länge des zurückgelegten Pfades, beginnend vom Startpunkt bis hin zum Konzept mit den letzten Änderungen/Löschungen/Erzeugungen, bildet somit eine Quasi-Metrik für den Lernfortschritt. Werden Instanzänderungen am Konzept des Endpunktes ermittelt, so kann davon ausgegangen werden, dass der Lernende den Prozess erfolgreich durchgebucht hat. Wurde der Pfad nur zur Hälfte durchschritten, kann auf Wissensdefizite geschlossen werden. In diesem Fall wäre dem Lernenden weiteres detailliertes Trainingsmaterial ab dem zuletzt erreichten Konzept nahezulegen. Gewichtungen 
der Übergänge ausgehend von den Instanz-Deltas und auch der zeitliche Dimension können in die Lernmetrik mitberücksichtigt werden. Neben der eigentlichen Fortschrittsanzeige, ist es durch den ET aber vor allem möglich, die Abhängigkeiten zwischen den einzelnen Systemkomponenten sichtbar zu machen und somit die Integration der einzelnen Bestandteile insbesondere hinsichtlich ihrer semantischen Perspektiven zu verdeutlichen.

\section{Zusammenfassung und Ausblick}

Ausgehend von der Bedeutung neuer Lernkonzepte für die wettbewerbsfähige Ausbildung an Hochschulen und Universitäten sowie den damit verbunden Problemen hinsichtlich Komplexität und Adaptivität, beschreibt dieser Beitrag ein Konzept für die Ausbildung an ERP-Systemen. Grundlage stellt dabei die Schaffung einer adaptiven Lernumgebung unter der Berücksichtigung neuer Technologien für die Auswertung großer betrieblicher Anwendungssysteme dar. Ziel dieses Ansatzes ist es, den Lernfortschritt am System zu dokumentieren und zu analysieren, um dem Lernenden die bestmögliche Unterstützung in seinem Ausbildungsprozess am System zu ermöglichen und dem Lehrenden Rückmeldungen zu dem aktuellen Fortschritt sowie eventuell auftretenden Probleme zu geben. Vorgestellt wurden hierzu zwei Verfahren, das AUM und die Enterprise Tomographie, die in Kombination eben genau dies ermöglichen: das Benutzerverhalten adaptiv zu steuern und dem Lernenden somit eine adaptive Lernumgebung gemäß seiner individuellen Voraussetzungen bereitzustellen.

In den nächsten Arbeiten werden die bereits existierenden prototypischen Entwicklungen zusammengeführt, um diese adaptive Lernumgebung vollständig zu realisieren und anschließend zu evaluieren. Des Weiteren wird die Methodik der didaktischen Unterstützung des Konzeptes verfeinert und hinsichtlich weiterer pädagogischer Aspekte erweitert, z. B. unter Einbeziehung von Web 2.0 Technologien.

\section{Literatur}

Aalmink J, Marx Gómez J (2009) Enterprise Tomography - an Efficient Approach for Semi-Automatic Localization of Integration Concepts in VLBAs. In: CruzCunha M M (Hrsg) Social, Managerial and Organi-zational Dimensions of Enterprise Information Systems. IGI Global, Hershey (PA), USA.

Ask U, Juell-Skielse G, Magnusson J, Olsen Dag H, Päivärinta T (2008) Enterprise Systems as Vehicles of Pedagogic Innovation - Enterprise System Inclusion in Higher Education. In: Proceedings of the 5th International Conference on Enterprise Systems, Accounting and Logistics (5th ICESAL '08), 7-8 July 2008, Crete Island, Greece. 
Brehm N, Haak L, Peters D (2009) Using FERP Systems to introduce web servicebased ERP Systems in Higher Education. In: Abramowicz W, Flejter D (Hrsg) Business Information Systems Workshops (BIS 2009), Poznan, Poland.

Courant J (2008) Beispiele für den Einsatz von SAP-Software in der Wirtschaftsinformatikausbildung im Kontext eines Lernmodells. In: Bichler M, Hess T, Krcmar H, Lechner U, Matthes F, Picot A, Speitkamp B, Wolf P (Hrsg) Tagungsband der Multikonferenz Wirtschaftsinformatik (MKWI 2008), München.

Freyburger K, Lehmann P (2008) Herausforderungen bei der Wirtschaftsinformatik Ausbildung mit Standardsoftware am Beispiel von mySAP Business Intelligence. In: Bichler M, Hess T, Krcmar H, Lechner U, Matthes F, Picot A, Speitkamp B, Wolf P (Hrsg) Tagungsband der Multikonferenz Wirtschaftsinformatik (MKWI 2008), München.

Hans D, Marx Gómez J, Peters D, Solsbach A (2008) Case study-design for Higher Education - a Demonstration in the Data Warehouse Environ-ment. In: Abramowicz W, Flejter D (Hrsg) Business Information Systems Workshops (BIS 2009), Poznan, Poland.

Kassem G, Marx Gómez J, Rautenstrauch C, Melato M (2005) Analysis of User's

Behaviour in Very Large Business Application Systems with Methods of the Web Usage Mining - A Case Study on SAP® R/3®. In: Menasalvas E, Segovia J, Szczepaniak P (Hrsg) Proceedings of 1st Atlantic Web Intelligence Conferences - Advances in Web Intelligence (AWIC 2003), Madrid.

Kassem G (2007) Application Usage Mining: Grundlagen und Verfahren. Shaker Verlag, Aachen.

Rautenstrauch C (2008) Wirtschaftsinformatik-Ausbildung mit SAP-Software. In: Bichler M, Hess T, Krcmar H, Lechner U, Matthes F, Picot A, Speit-kamp B, Wolf P (Hrsg) Tagungsband der Multikonferenz Wirtschafts-informatik (MKWI 2008), München.

St. Pierre R (2007) Educational Video Game Design: a Research/Design Methodology. Dissertation at the University of Quebec at Montreal (UQAM).

Wolf K (2007) Die Berücksichtigung des Gender-Mainstreaming-Ansatzes bei der Gestaltung von E-Learning Angeboten. In Hochschule und Weiter-bildung, 1, 2007, S. 69-78. 